Nivel de aplicación de estrategias y herramientas de administración en las PYMES hoteleras de Manabí

\title{
Nivel de aplicación de estrategias y herramientas de administración en las PYMES hoteleras de Manabí
}

\section{Level of application of strategies and administrative tools in the hotel smes of Manabí}

\section{Nível de aplicação de estratégias e ferramentas de administração no hotel PYMES de Manabí}

\author{
Johnny E. Ponce-Andrade I \\ johnny.ponce@uleam.edu.ec \\ Pedro J. Quijije-Anchundia II \\ pedro.quijije@uleam.edu.ec \\ Carlos A. Álvarez-Cárdenas III \\ carlos.alvarez@uleam.edu.ec \\ Claudia A. Molina-Quiroz ${ }^{\text {IV }}$ \\ analia.molina@uleam.edu.ec
}

Recibido: 15 de septiembre de 2017 * Corregido: 18 de octubre de 2017 * Aceptado: 10 de diciembre de 2018

I. Magister en Dirección Estratégica de Marketing y Ventas, Doctorante, Especialista en Gerencia Estratégica de la Comunicación Organizacional, Diploma Superior en Educación Universitaria por Competencias, Ingeniero Comercial, Docente de la Universidad Eloy Alfaro de Manabí, Jipijapa, Ecuador.

II. Magister en Gerencia de Proyectos Educativos y Sociales, Doctorante, Especialista en Diseño Curricular por Competencia, Doctor en Ciencias de la Educación mención Investigación y Planificación Educativa, Licenciado en Ciencias de la Educación Especialización Psicopedagogia y Técnicas de la Enseñanza, Profesor de Segunda Enseñanza Especialidad Psicopedagogia y Técnicas de Enseñanza, Docente Universidad Eloy Alfaro de Manabí, Jipijapa, Ecuador.

III. Magister en Dirección Estratégica de Marketing y Ventas, Doctorante, Abogado de los Juzgados y Tribunales de la Republica, Licenciado en Ciencias Sociales y Políticas, Docente Universidad Eloy Alfaro de Manabí, Jipijapa, Ecuador.

Iv. Magister en Administración Publica mención Desarrollo Institucional, Doctorante, Economista, Docente de la Universidad Eloy Alfaro de Manabí, Jipíjapa, Ecuador. 


\title{
Resumen
}

En esta era de la gestión del conocimiento y el desarrollo tecnológico, resulta imperante para las organizaciones de todo tamaño tener definidas sus estrategias empresariales; si una empresa quiere asegurar su supervivencia en el tiempo, debe tener un plan estratégico corporativo definido, con medidas y uso de recursos para alcanzar sus metas; así la gestión comercial se transforma hoy en una de los aspectos más angulares en una organización, que aplica la administración moderna para lograr su crecimiento. Este estudio devela como están los niveles de aplicación de los elementos en mención en las PYMES hoteleras de Manabí. Se aplicó el método lógico inductivo completo, con validación de hipótesis, implementando el software estadístico SPSS 21, con prueba de Alfa de Cronbach para medir la confiabilidad del instrumento constructo de investigación. Sobre una base de 246 encuestas aplicadas a gerentes/administradores de este sector y tipo de empresas se levantaron los datos.

Palabras clave: estrategia; PYME; gestión comercial; crecimiento empresarial; turismo.

\begin{abstract}
In this era of knowledge management and technological development, it is imperative for organizations of all sizes to have defined their business strategies; if a company wants to ensure its survival over time, it must have a defined corporate strategic plan, with measures and use of resources to achieve its goals; thus, commercial management is today transformed into one of the most angular aspects in an organization, which applies modern administration to achieve its growth. This study reveals how are the application levels of the elements in mention in the hotel PYMES of Manabí. The complete inductive logic method was applied, with hypothesis validation, implementing the statistical software SPSS 21, with Cronbach's Alpha test to measure the reliability of the research construct instrument. On a base of 246 surveys applied to managers / administrators of this sector and type of companies, the data was collected.
\end{abstract}

Keywords: strategy; SMEs; commercial management; Business Growth; tourism.

\section{Resumo}

Nesta era de gestão do conhecimento e desenvolvimento tecnológico, é imperativo que organizações de todos os tamanhos tenham definido suas estratégias de negócios; se uma empresa quiser garantir a sua sobrevivência ao longo do tempo, deve ter um plano estratégico corporativo definido, com medidas e uso de recursos para atingir seus objetivos; Assim, a gestão comercial é hoje transformada 
em um dos aspectos mais angulares de uma organização, que aplica a administração moderna para alcançar seu crescimento. Este estudo revela como são os níveis de aplicação dos elementos mencionados no hotel PYMES de Manabí. O método da lógica indutiva completa foi aplicado, com validação de hipóteses, implementando o software estatístico SPSS 21, com o teste Alpha de Cronbach para medir a confiabilidade do instrumento de construção de pesquisa. Com base em 246 pesquisas aplicadas a gerentes / administradores deste setor e tipo de empresas, os dados foram coletados.

Palavras chave: estratégia; PME; gestão comercial; crescimento do negócio; turismo

\section{Introducción}

El curso del futuro de toda empresa se centrara en tener definido como primer enfoque estratégico sus postulados corporativos, tales como la misión, visión, valores y objetivos. En principio seria la demostración cierta de que existe una hoja de ruta empresarial, para ir desarrollando las actividades diarias que apunten al logro de las metas.

Sin embargo, también es necesario el manejo y aplicación de herramientas modernas de la administración, en vínculo pleno con la tecnología, para ello el empresario PYMES, debe contar con los conocimientos adecuados para la dirección empresarial, y en el caso de no tenerlos, lo importante es que debe ampliar su pensamiento de forma holística; necesita contratar personal de alto nivel, para que mejore la competitividad y la gestión en su organización, en lo que bien podríamos llamar inteligencia de negocios (Business Intelligence).

Las PYMES del sector hotelero de la provincia de Manabí-Ecuador, que son el objeto de estudio de esta investigación, no pueden estar exentas de estos enfoques y orientaciones, de lo contrario le estarían restando efectividad a su accionar, en un sector muy sensible y crítico en los actuales momentos, el cual aún acarrea las secuelas del terremoto del 16 de abril del 2016, que ha minado de a poco la situación económica de la pequeñas y medianas empresas de hospedaje, sumándose hoy la recesión económica del país, que tiene en vilo al empresario manabita en particular.

Una PYMES hotelera tiene como característica principal el otorgamiento de servicios de hospedaje y sus conexos (tours, restaurante, piscina, movilización, bar, discoteca, garaje, etc.) dependiendo de su tamaño, referenciado que en el Ecuador existen clasificaciones en los distintos tipos de empresas; estas organizaciones manejan productos intangibles, donde su principal fortaleza se debe centrar en la 
gestión comercial y la calidad de atención al cliente, con todas sus estrategias para incentivar el consumo turístico, aprovechando los atractivos de Manabí como destino.

En las organizaciones de servicios, una de las funciones más importantes es la gestión comercial (GC), cuya tarea es la de encargarse de la apertura de la empresa al mercado, con muchas labores por enfrentar, siendo principalmente la más ardua, el reto de lograr la satisfacción al cliente, alcanzar un buena participación de mercado, incrementar las ventas, desdoblar las actividades del marketing, preparar al recurso humano, optimizar procesos administrativos, entre otras, alineando en este acople de tareas el logro de la rentabilidad, que encamina el crecimiento empresarial.

Citando a Herrero (2011), respecto a la gestión comercial, la define como "la actividad que se ocupa de la relación de intercambio de la organización con el mercado" (p.63). Ugarte (2003), señala "la comercialización es el conjunto de las acciones encaminadas a comercializar productos, bienes o servicios. Las técnicas de comercialización abarcan varios procedimientos para introducir eficazmente los productos en el sistema de distribución" (p.32).

Debe estar claro que las funciones de la GC, no solo solamente están ligadas a la venta, pues su ámbito de acción es mucho más vasto, ya se ha mencionado que no solo se encarga de estudiar el mercado, sino de la distribución misma del producto y/o servicio, colocándolo a disposición del consumidor final, siendo imperante la implementación de estrategias, sobre todo comerciales, con políticas y procedimientos de carácter interno (empresa), como para los clientes, denotándose lamentablemente una acentuada ausencia en gran cantidad de pequeñas y medianas empresas del presente estudio.

Con marcadas diferencias respecto a las PYMES, las grandes empresas tienen normados numerosos procesos de la administración, como la planificación estratégica y la toma de decisiones, mismos que afligen al desempeño de la gestión comercial en unidades de negocios pequeñas.

Como resultado propio de la definición de objetivos, fijados a un máximo nivel organizativo en la gran empresa, contrariamente en las PYMES la falta de una estructura orgánica/administrativa, la gestión comercial pierda su fuerza estratégica, dado que las organizaciones deben centrar su gestión en la búsqueda de soluciones operativas, y no en los enfoques estratégicos de la GC, tal como se ilustra en el siguiente figura: 
Figura 1: Enfoques estratégicos para la gestión comercial (GC)

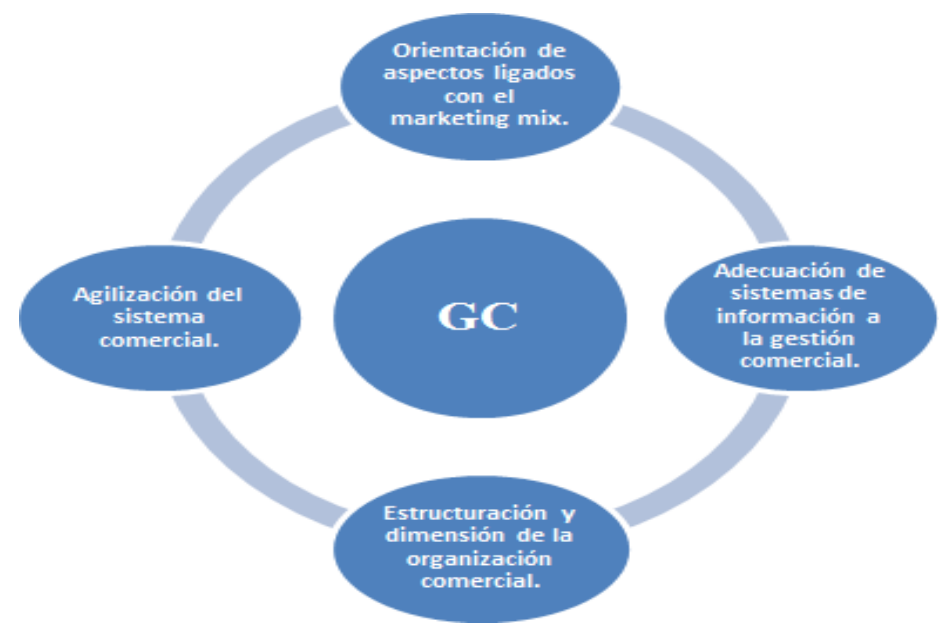

Fuente: Adaptación propia de autores

Desafortunadamente en la mayoría de las PYMES estudiadas, el proceso de la planificación estratégica y la gestión comercial no se institucionaliza, en ocasiones por la falta de conocimientos, de iniciativa, entre otros factores, sin tomar en cuenta la importancia que tienen estos componentes para su desarrollo, dado que este tipo de organizaciones debe asumir un doble rol a nivel de la planeación comercial y la mercadotécnica, de donde se plantean los objetivos y decisiones empresariales más importantes, ya que no hay empresa que pueda subsistir sin la comercialización de sus productos/servicios. El proceso del planeamiento estratégico, obliga también a preparase para dar respuesta a los problemas operativos de la venta y el marketing, que al igual que en las grandes compañías, son propias de las tácticas que se adopten, como parte de la gestión sistémica corporativa.

En el tiempo han sido muy escazas las investigaciones realizadas en torno a las PYMES hoteleras en la provincia de Manabí, y más en una temática como la aplicación de estrategias y el uso de herramientas de la administración, donde la gestión comercial es la dimensión más significativa para lograr el crecimiento de este tipo de empresas, en un sector como ya se manifestó, está viviendo los efectos post desastre del terremoto de 7.8 grados en la escala Richter en abril del 2016.

\section{Dificultades y ambiente de las PYMES de Manabí}

Las pequeñas y medianas empresas hoteleras, son un eje sustancial en la economía de la región, por su tributo al impulso económico y al bienestar social de la población, generando un alto aporte al 
crecimiento y la producción regional de servicios, con evidente contribución para el país; son agentes económicos generadores de un importante porcentaje de empleos directos e indirectos en cada cantón, cubriendo una parte significativa de la demanda de productos/servicios en la provincia.

De acuerdo a datos levantados en el Instituto Nacional de Estadísticas y Censos (INEC), la base de datos del Directorio de Empresas y Establecimientos (DIEE) 2016, en el Ecuador existen un total de 843.745 empresas en sus distintos tamaños, de las cuales 763.636 que equivalen al $90.51 \%$ son microempresas albergando de 1 a 9 trabajadores; 63.400 son consideradas como pequeñas, que corresponden al 7.51\%, las mismas que ocupan entre 10 a 49 personas; 7.703, es decir el 0.91\% están clasificadas como medianas "A", con un total de 50 a 99 personas contratadas, y 5.143 unidades de negocios, con el 0,61\%, manejando de 100 a 199 trabajadores, son catalogadas como "B", tal como se lo ilustra en el siguiente gráfico.

Figura 2: Clasificación y tamaño de las empresas en Ecuador

\section{Grande:}

- V: $\$ 5$ '000.001 en adelante. P: 200 en adelante.

\section{Mediana "B":}

- V: \$2'000.001 a \$5’000.000. P: 100 a 199.

\section{Mediana "A"}

- V: \$1'000.001 a \$2’000.000. P: 50 a 99.

\section{Pequeña:}

- V: $\$ \$ 100.001$ a $\$ 1^{\prime} 000.000$. P: 10 a 49

\section{Microempresa:}

- V: < a \$100.000. P: 1 a 9.

Fuente: INEC - DIEE 2016 
Figura 3: Estructura según el tamaño de las empresas en Ecuador

\begin{tabular}{|c|c|c|c|}
\hline $\begin{array}{c}0,46 \% \\
0,61 \% \\
0,91 \%\end{array}$ & $90,51 \%$ & $\begin{array}{l}\text { | Microempresa } \\
\text { | } \text { Pequeña empresa } \\
\text { |" Mediana empresa "A" } \\
\text { | Mediana empresa "B" } \\
\text { " Grande empresa }\end{array}$ & \\
\hline Tamaño de Empresa 2016 & & No. Empresas & Porcentaje \\
\hline Microempresa & & 763.636 & $90,51 \%$ \\
\hline Pequeña empresa & & 63.400 & $7,51 \%$ \\
\hline Mediana empresa "A" & & 7.703 & $0,91 \%$ \\
\hline Mediana empresa "B" & & 5.143 & $0,61 \%$ \\
\hline Grande empresa & & 3.863 & $0,46 \%$ \\
\hline Total & & 843.745 & $100,00 \%$ \\
\hline
\end{tabular}

Fuente: INEC - DIEE 2016

Por otra parte, investigaciones realizadas por instituciones como la Universidad Laica Eloy Alfaro de Manabí (ULEAM), revelan que el $72 \%$ de las pequeñas y medianas empresas de todo sector y subsector (76.246), no gastan en capacitación y tan solo el $28 \%$ asignan recursos para este propósito.

En esa medida, y para efectos ceñidos al ámbito estudiado (hotelero), se identifican problemas como la falta de manejo del idioma universal de los negocios (inglés) y otras lenguas, indispensables para la relación de comunicación que se genera en la actividad estudiada, donde los huéspedes pueden resultar de distintas nacionalidades; esta carencia hace que las pequeñas y medianas empresas hoteleras de Manabí, pierdan permanentemente consumidores, por la incomprensión del idioma y el no saber promocionar las bondades de sus servicios. Se adicionan otras complejidades, como la impericia de una atención especializada al cliente, imprescindible en el turismo, se agregan a la par la falta de conocimientos para tener fluidos procesos administrativos, contables, tecnológicos, y otros, conspirando al escenario de una disminuida competitividad, por la poca capacitación al personal, 
donde está inmerso el propio empresario/propietario/administrador, el cual al no tener un claro horizonte del negocio, se transforma en parte del problema.

Las empresas que no se encauzan en la aplicación de un modelo estratégico de negocios, aprovechando las herramientas de la administración moderna, corren un alto riesgo de desaparecer. El estudio realizado por la ULEAM, muestra que existe un alto índice de mortalidad de las PYMES regionales y nacionales. Los números de fracaso son abrumadores, estadísticamente se evidencia que en promedio el $80 \%$ perecen antes de los cinco años, y el $90 \%$ de ellas no llegan a los diez, pudiéndose determinar las principales dificultades, contra las que tienen que batallar estas empresas, develándose problemas para sostenerse en el mercado por su deleznable crecimiento, originado por la falta de una gestión orientada al desarrollo y aplicación de estrategias de comercialización, transformándose en una obstáculo para desarrollar su cartera de productos y/o servicios. El uso de estrategias y tácticas de marketing es imprescindible en el desdoblamiento de la gestión comercial, dado que permite visualizar el contexto de las 4 variables (producto, precio, plaza y promoción), sobre la cual giran las decisiones más importantes en una empresa, debiendo saber si existe o no una propuesta atractiva para los clientes, con un precio adecuado, con canales de distribución idóneos, promocionados por la vía adecuada. Si no se gestiona bajo estos enfoques a una organización, se castiga la venta, la participación de mercado y ende el crecimiento, con el riesgo de extinguirse.

Justamente, respecto a aplicación de la gestión comercial y el marketing en las PYMES del sector investigado, se ha podido comprobar que están en una etapa inicial, cuyo desconocimiento no les permite crear de forma técnica sus productos, tampoco trabajan en el posicionamiento de la marca/empresa, sobrellevándolas a no alcanzar ventajas comparativas y competitivas, más que todo de diferenciación, dada la informalidad en la entrega de sus intangibles. Las pequeñas y medianas empresas hoteleras Manabitas se preocupan más por su flujo de caja diario, debiendo aprender a fortalecer la variable mencionada y sus dimensiones, esenciales para su desarrollo y supervivencia.

Otro aspecto donde deben lograr un mejor desenvolvimiento, es en la atención al cliente, articulando una práctica apropiada de servucción, y no lo que se evidencia, una desprovista calidad del servicio.

Para tener una clara idea de lo que busca la servucción, referenciamos en parafraseo la conceptualización realizada por los educadores Pierre Eiglier y Eric Langeard (1989), de acuerdo a 
publicación analizada de Gustavo Alonso (2008). Desde el perspectiva del marketing estratégico, la servucción, es la articulación de los elementos humanos y físicos (exposición del lugar de trabajo), que permiten construir una armónica relación entre el cliente y la empresa, forjando un ambiente adecuado para la prestación de los servicios, y así demostrar por medio de la calidad, cuan satisfechos pueden quedar los clientes. Se reitera entonces que una empresa de cualquier tamaño que no trabaja bajo una línea de gestión estratégica y comercial, tiene casi asegurado su fracaso.

Los problemas que se generan en la comercialización, dependen también en buena medida del recurso humano, el mismo que de acuerdo a los datos recabados, no es del mejor nivel profesional, en muchos casos sin experiencia y carente de capacitación. La mayoría de PYMES son familiares, con una estructura orgánica-administrativa vertical, donde poder de decisión lo tiene una sola persona; dada su visión de corto plazo, no se contrata personal competente, tampoco se aplica un esquema de incentivos, lo cual desmotiva al personal, especialmente al del área de comercial.

En función de lo analizado es importante citar a Chiavenato (2011), quien expresa que "la gestión del talento humano en las organizaciones es la función que permite la colaboración eficaz de las personas [empleados, funcionarios, recursos humanos en general] para alcanzar los objetivos organizacionales e individuales". (p.95).

El PYME hotelero de Manabí, debe formarse para aprender a utilizar los recursos y herramientas que provee la administración moderna, y así por ejemplo conocer cuáles son las necesidades presentes y proyectar las futuras de sus consumidores; ciertamente este es un reto a sortear en los actuales momentos para el pequeño y mediano empresario, el cual debe imperiosamente aplicar estrategias comerciales y de marketing en su negocio, orientadas desde una base de información, sobre las motivaciones y características del consuno de sus productos y/o servicios, partiendo desde un buen estudio de mercado, sin que se considere una inversión sin retorno, y no un gasto como lo admiten la mayoría de organizaciones grandes en su contabilidad. Por este motivo, cuando surgen nuevos contendientes en el mercado, no les proporcionan la atención debida, cuya consecuencia de una reacción tardía, es la pérdida de participación de mercado, causado por el bajo nivel de sus ventas.

Va quedando claro entonces que parte del problema pasa por la informalidad, con que se llevan los procesos de administración en las PYMES, y se reitera que cierta toma de decisiones tardías ocurren 
porque estas organizaciones son familiares, donde las acciones estratégicas y operativas mal desdobladas, producto de una visión cortoplacista, no les permite explotar apropiadamente las fortalezas geográficas que brinda Manabí como destino turístico, con extendidas playas, un prodigioso patrimonio histórico y cultural, gustosa gastronomía, centros comerciales en los cantones más importantes de la región, sumándose aquellos Stakeholders a favor del sector hotelero, como agencias de viajes, operadores turísticos, compañías de transporte terrestre, marítimo, aéreo, entidades gubernamentales como el Ministerio de Turismo, Cámara de Turismo, Direcciones de Turismo de los Gobiernos Autónomos Descentralizados y Gobierno Provincial de Manabí, lo que favorece a la demarcación de diferencias con empresas de servicios hoteleros de otros territorios.

Sin embrago, el problema más complejo de todo el contexto descrito, es el de afrontar aun los efectos del terremoto sufrido el 16 de abril del 2016, justo con epicentro en Manabí. Este acontecimiento sin precedentes históricos, cambio la vida de la población, de sus entidades gubernamentales y de las organizaciones privadas a todo nivel, puesto que este desastre natural ocasiono una crisis productiva, psicológica y anímica en el los ciudadanos no solo de la región, sino del Ecuador y el mundo, afectando hasta hoy terriblemente el consumo del producto turístico, más aún en una PYME que tuvo que observar como parte de su infraestructura se vio casi devastada.

El análisis realizado hasta ahora, admite deducir de forma muy reflexiva, que en este momento las PYMES de esta provincia del Ecuador, necesitan realizar mejoras a la gestión comercial de sus empresas, utilizando al marketing como un instrumento consistente para alcanzar las metas de recuperación del sector. Por ello, si las pequeñas y medianas empresas Manabitas no sorprenden a sus consumidores, con propuestas que no efectúa la competencia, no existirá ninguna posibilidad de sostenerse en la industria turística; Philip Kotler (2005), propone en la siguiente cita que "se debe conseguir y mantener [fidelizar] al cliente, fijándole valores a la empresa, con objetivos y visión de futuro" (p.12).

\section{Marco conceptual del estudio}

La visión empresarial toma hoy un giro diametralmente global, se vive época de incesantes y acelerados cambios, un momento económico diferente, con toma de decisiones rápidas e inteligentes, 
donde es necesario cambiar el paradigma de las organizaciones por uno superior. Históricamente estos enunciados se apoyan en concepciones ilustradas por Russell Ackoff (1979), filósofo norteamericano que anima a pasar la fase denominada como la era de las maquinas. Empieza entonces la empresa a transformarse en un sistema social, con planes y proyectos propios, para formar parte de sistemas más desarrollados, con tipologías parecidas a la de los centros de educación superior, de investigación científica, tecnológica y de gobiernos de diferentes alcances, con el fin de generar I+D.

A escala planetaria, el modelo de gestión empresarial y comercial ha cambiado su estrategia, yendo desde el trabajo en equipo, hasta el uso de herramientas tecnológicas como puntal de la gestión humana, permitiendo demostrar que la configuración transaccional era incapaz de proporcionar respuestas que satisfagan los inconvenientes comerciales pasados, que no decir de la relevancia que viene tomando la mercadotecnia en todos estos cambios.

Siendo la gestión comercial y el crecimiento, las variables más importantes de esta investigación, donde se analizan varias dimensiones, es imperante generar las bases epistemológicas de las mismas, citando a varios teóricos.

Herrero (2011) expresa que "la gestión comercial, es la que lleva a cabo la relación de intercambio de la empresa con el mercado" (p.151).

Borja de Carlos Martín-Lagos, Francisco (2008), expone que "la gestión comercial forma parte esencial del funcionamiento de las organizaciones: decisiones relativas a que mercados acceder; con que productos, que política de precios aplicar; como desarrollar una sistemática comercial eficaz. Además de ser aspectos que emanan directamente de las decisiones derivadas de la estrategia corporativa". (pp.9 y 10).

Teóricos como David Ricardo (2013), indican que el crecimiento económico y el comercio, han sido variables estudiadas dentro de la orientación de la filología económica, en supuestos institucionales de la economía de mercado plenamente competitiva. De acuerdo a esta visión, tan solo las organizaciones y los estados compiten; en los años setenta, dada la progresiva competitividad en el mercado mundial, como resultado de las regulaciones que rigen las relaciones universales y los cambios en la tecnología, se ha ido transformando el contexto corporativo e institucional en que está inmerso el crecimiento y el comercio. 
Refiere Krugman (2010), respecto al crecimiento de las organizaciones, que estas evidencian modificaciones e incrementos de tamaño, originando una diferencia con su estado anterior. Esto significa que se ha producido un incremento tanto en cantidades (unidades de producción) como en dimensión (infraestructura física), como también variaciones en sus características internas (estructura económica y organizativa). Visto de otra manera, los aumentos se pueden comprobar en todas o varias de las subsecuentes variables: activos, producción, ventas, beneficios, líneas de productos, mercados, recurso humano, etc.

\section{Hipótesis de la investigación}

Se referencio que era necesario estructurar un ámbito de análisis, desde dos variables de estudio (gestión comercial y crecimiento) con varias dimensiones (tres por variable: prácticas de marketing, programas de capacitación, innovación de procesos administrativos, participación de mercado, ventas y rentabilidad), las mismas que permitan delimitar de manera precisa los aspectos de evaluación y medición, relacionados al tema del artículo, como es el nivel de aplicación de estrategias y herramientas de administración en las PYMES del sector hotelero de Manabí. Toda investigación de carácter científico, conlleva el planteamiento de hipótesis, por ello se trazó una general y tres específicas, tal como se detalla:

General: La gestión comercial incide en el crecimiento de las PYMES del sector hotelero de Manabí.

\section{Específicas:}

1. Las prácticas del Marketing inciden en el crecimiento de las PYMES del sector hotelero de Manabí.

2. Los programas de capacitación para el RRHH, inciden en el crecimiento de las PYMES del sector hotelero de Manabí.

3. La innovación de los procesos administrativos, inciden en el crecimiento de las PYMES del sector hotelero de Manabí.

\section{Mapa de variables}


Dom. Cien., ISSN: 2477-8818

Vol. 4, núm. 1, Enero, 2017, pp. 206-234

Nivel de aplicación de estrategias y herramientas de administración en las PYMES hoteleras de Manabí

\begin{tabular}{|c|c|c|c|}
\hline Variables & Dimensiones & Indicadores & Ítems \\
\hline \multirow{3}{*}{$\begin{array}{c}\text { X. Gestión Comercial } \\
\text { (V.Independiente) }\end{array}$} & $\begin{array}{l}\text { Prácticas de Marketing } \\
\qquad(4 \mathrm{P}) \text {. }\end{array}$ & $\begin{array}{l}\text { Realización de actividades } \\
\text { relacionadas con el } \\
\text { Marketing. }\end{array}$ & $1-2-3-4-5$ \\
\hline & $\begin{array}{l}\text { Programas de capacitación } \\
\text { del RR.HH. }\end{array}$ & $\begin{array}{l}\text { Mejora del rendimiento } \\
\text { económico fruto de } \\
\text { capacitaciones. }\end{array}$ & $6-7-8-9-10$ \\
\hline & $\begin{array}{l}\text { Innovación de los procesos } \\
\text { administrativos. }\end{array}$ & $\begin{array}{l}\text { Cambios administrativos } \\
\text { con agregación de equipos } \\
\text { tecnológicos. }\end{array}$ & $11-12-13-14-15$ \\
\hline
\end{tabular}

\begin{tabular}{|l|l|l|c|}
\hline \multirow{2}{*}{ Y. Crecimiento } & Participación de mercado. & $\begin{array}{l}\text { Medición del avance de } \\
\text { las ventas. }\end{array}$ & 16-17-18-19-20 \\
\cline { 2 - 4 } (V.Dependiente) & Ventas. & $\begin{array}{l}\text { Crecimiento de la cartera } \\
\text { de clientes/turistas por } \\
\text { aceptación de servicios. }\end{array}$ & $21-22-23-24-25$ \\
\cline { 2 - 4 } & Rentabilidad. & $\begin{array}{l}\text { Informede resultados para } \\
\text { establecer pérdidas }\end{array}$ & $26-27-28-29-30$ \\
\hline
\end{tabular}

\section{Metodología}

Se utilizo la metodologia de investigacion lógica inductiva completa, la cual de acuerdo afirmación de Roberto Hernández Sampieri y Carlos Fernández Collado (2010), es el razonamiento que parte de casos particulares y se eleva a conocimientos generales. Es válido a través de este método plantear hipótesis y estudiar leyes científicas con sus pertinentes demostraciones. Tomando referencia de Pablo Cazau (2006), para el alcance del presente estudio se utilizó la investigación exploratoria, descriptiva, correlacional y explicativa, manteniendo fases cronológicas de todo el proceso investigativo, puesto 
que cada tipología tiene un fin y tiempo distinto. De esta manera lo que se procuró en primera instancia es explorar y profundizar en el tema, después detallar las variables involucradas, subsiguientemente correlacionarlas entre sí, finalmente explicar la influencia de una variable sobre la otra en términos de causalidad.

\section{Investigación mediante encuesta}

Este es un instrumento válido y efectivo de medición, para levantar información de corte cuantitativo, a través de un sistema de encuesta con ítems en escala de Likert, cuyo esquema de carácter no experimental no transversal, como lo determinan Donald T. Campbell y Julián C. Stanley (1978), “toma la información en un momento determinado del tiempo, para obtener datos relevantes y sustanciales de la población objetivo" (p.40), leído en publicación de R. Hernández Sampieri y C. Fernández Collado (2010). El uso de esta técnica hizo posible conocer el problema de las PYMES y el sector desde la óptica del gerente/administrador.

\section{Unidad de análisis}

La investigación se efectuó en las PYMES del sector hotelero de la provincia de Manabí, con un universo de 686 unidades de acuerdo a su formalidad legal (constituidas), con fuente informativa del Instituto Nacional de Estadísticas y Censos (INEC), Directorio de Empresas y Establecimientos (DIEE) y el SRI.

\section{Tamaño de la muestra}

Para establecer el tamaño de la muestra se empleó el muestreo probabilístico, el cual permite medir el error, tratando de minimizar el mismo de acuerdo a Kish, Kalton y Heeringa (2003), como también conocer el nivel de confianza, utilizando la siguiente fórmula estadística.

$$
n=\frac{Z^{2} \cdot P \cdot Q \cdot N}{Z^{2} \cdot P \cdot Q+(N)(e)^{2}}
$$

Reemplazados los datos de la fórmula, se obtuvo una muestra de 246 PYMES hoteleras, de las cuales se estudiaron 100 pequeñas y 146 medianas empresas, entre tipo A y B en los distintos cantones donde se aplicaron las encuestas, tomando en cuenta la siguiente distribución: 
Tabla 1: Distribución de las encuestas por tipo de empresa

\begin{tabular}{|c|l|c|c|c|c|}
\hline & \multicolumn{1}{|c|}{ Tipo } & Frecuencia & Porcentaje & Porcentaje válido & $\begin{array}{c}\text { Porcentaje } \\
\text { Acumulado }\end{array}$ \\
\hline \multirow{4}{*}{ Válidos } & Pequeña & 100 & 40,7 & 40,7 & 40,7 \\
\cline { 2 - 6 } & Mediana-A & 73 & 29,7 & 29,7 & 70,3 \\
\cline { 2 - 6 } & Mediana -B & 73 & 29,7 & 29,7 & 100,0 \\
\cline { 2 - 6 } & Total & 246 & 100,0 & 100,0 & \\
\hline
\end{tabular}

Fuente: Encuestas aplicadas a las PYMES hoteleras de Manabí - Análisis Software SPSS 21

Tabla 2: Distribución de las encuestas por Cantones

\begin{tabular}{|c|l|c|c|c|c|}
\hline \multirow{1}{*}{ Cantón } & Frecuencia & Porcentaje & Porcentaje válido & $\begin{array}{c}\text { Porcentaje } \\
\text { Acumulado }\end{array}$ \\
\hline \multirow{5}{*}{} & Manta & 72 & 29,3 & 29,3 & 29,3 \\
\cline { 2 - 6 } & Portoviejo & 50 & 20,3 & 20,3 & 49,6 \\
\cline { 2 - 6 } & Sucre & 26 & 10,6 & 10,6 & 60,2 \\
\cline { 2 - 6 } & San Vicente & 23 & 9,3 & 9,3 & 69,5 \\
\cline { 2 - 6 } & Jama & 10 & 4,1 & 4,1 & 73,6 \\
\cline { 2 - 6 } & Pedernales & 32 & 13,0 & 13,0 & 86,6 \\
\cline { 2 - 6 } & El Carmen & 4 & 1,6 & 1,6 & 88,2 \\
\cline { 2 - 6 } & Chone & 3 & 1,2 & 1,2 & 99,4 \\
\cline { 2 - 6 } & Jipijapa & 6 & 2,4 & 2,4 & 98,8 \\
\cline { 2 - 6 } & Puerto López & 17 & 6,9 & 6,9 & 100,0 \\
\cline { 2 - 6 } & Santa Ana & 3 & 1,2 & 1,2 & \\
\cline { 2 - 6 } & Total & 246 & 100,0 & 100,0 & \\
\hline
\end{tabular}

Fuente: Encuestas aplicadas a las PYMES Turísticas Hoteleras de Manabí - Análisis Software SPSS 21

\section{Técnicas de recolección de datos}

Se corrieron 246 encuestas, respondidas por sus gerentes/administradores. El instrumento original (validado por expertos PhD) está conformado por 30 ítems, 15 por cada variable (5 por cada dimensión, con relación directa a indicadores), con calificación de hasta 5 puntos, de acuerdo al siguiente orden: nunca (equivale a 1), muy pocas veces (equivale a 2), algunas veces (equivale a 3), casi siempre (equivale a 4), siempre (equivale a 5). 


\section{Resultados}

Utilizando el software estadístico SPSS 21, se procesó el banco de preguntas sobre las variables gestión comercial y crecimiento, con sus respectivas dimensiones, realizándose el análisis de fiabilidad y validación del instrumento de medición por medio del Alfa de Cronbach. Este coeficiente determina que mientras el valor calculado sea más cercano a uno (1), mayor será la confiabilidad del instrumento. El resultado se detalla en la siguiente tabla.

Tabla 3: Fiabilidad Alfa de Cronbach

\begin{tabular}{|c|c|}
\hline Alfa de Cronbach & N de elementos \\
\hline, 994 & 30 \\
\hline
\end{tabular}

Fuente: Análisis Software SPSS 21

La tabla 3, muestra que el coeficiente de fiabilidad del Alfa de Cronbach es de 0,994 por consistencia para los 30 ítems, por tanto el existe una excelente fiabilidad.

\section{Prueba de hipótesis}

Utilizada la encuesta con opciones de tipo Likert con cinco categorías de respuesta, se aclara que las categorías no implican ninguna ordenación, simplemente identifican al elemento, por tanto las escalas de medida de las variables son nominales. Así, se realizaron pruebas no paramétricas bivariadas, mediante el análisis de correlación de Spearman para la comprobación de las hipótesis en el software estadístico SPSS 21. Para interpretar los resultados se toma en cuenta el baremo de correlación para la interpretación de prueba de hipótesis, expresado de la siguiente manera: Si el coeficiente está entre 0 y 0,2 , entonces la correlación es mínima; si está entre 0,2 y 0,4 , es una correlación baja; si se encuentra entre 0,4 y 0,6, entonces es una correlación moderada, si está entre 0,6 y 0,8 es una correlación buena; y si se encuentra entre 0,8 y 1, es una correlación muy buena. Esto mismo aplica en negativo. Así los resultados obtenidos se despliegan en la tabla a continuación detallada: 
Tabla 4: Resumen de prueba de hipótesis

\begin{tabular}{|c|c|c|c|c|c|}
\hline Hipótesis & $\begin{array}{c}\text { Variable } \\
\text { Independiente }\end{array}$ & $\begin{array}{c}\text { Variable } \\
\text { Dependiente }\end{array}$ & Sig & $\begin{array}{l}\text { Rho de } \\
\text { Spearman }\end{array}$ & Resultado \\
\hline General & X. Gestión Comercial & \multirow{4}{*}{$\begin{array}{l}\text { Y. Crecimiento } \\
\text { de las PYMES. }\end{array}$} &, 000 & ,966 & Se rechaza Ho \\
\hline Especifica 1 & $\begin{array}{l}\text { X1. Practicas de } \\
\text { Marketing. }\end{array}$ & &, 000 & ,959 & Se rechaza Ho \\
\hline Especifica 2 & $\begin{array}{l}\text { X2. Programas de } \\
\text { capacitación para el } \\
\text { RR.HH. }\end{array}$ & &, 000 & ,974 & Se rechaza Ho \\
\hline Especifica 3 & $\begin{array}{l}\text { X3. Innovación de los } \\
\text { procesos } \\
\text { administrativos. }\end{array}$ & &, 000 & ,957 & Se rechaza Ho \\
\hline
\end{tabular}

Fuente: Resumen luego de análisis del Software SPSS 21

Elaboración: Autores.

Al analizar la tabla 4, de acuerdo al programa estadístico, se establece que existe un nivel de correlación alta en la comprobación de la hipótesis general $(0,966)$, puesto que está mucho más cercano a 1, de acuerdo a lo que determina el baremo de medición. Por otro lado, se establece que es una correlación positiva, es decir, a mayor nivel de "Gestión Comercial", mayor será el crecimiento de las PYMES, por lo tanto, se cumple lo que se planteó al escoger las variables. Al analizar la significación, vemos que es de 0,000, nuevamente se cumple que la condición de que el grado de significancia sea menor a 0,05 , incluso es menor a 0,01 , lo que indica que la correlación que se ha establecido es cierta. En consecuencia, se rechaza la hipótesis nula y se acepta la hipótesis alternativa.

En la comprobación de la hipótesis especifica 1, se establece la existencia de un nivel de correlación alta $(0,959)$, siendo que está mucho más cercano a 1 . Por lo tanto es una correlación positiva, es decir, a mayor nivel de "Prácticas de Marketing", mayor será el crecimiento de las PYMES, cumpliéndose con lo que se planteó al escoger las variables. Vemos que la significación es de 0,000, se cumple también con la condición de que el grado de significancia sea por debajo de 0,05 , incluso es menor a 0,01, lo que demuestra que la correlación que se ha establecido es cierta. Por consiguiente, se rechaza la hipótesis nula y se acepta la hipótesis alternativa. 
Comprobando la hipótesis especifica 2, se determina un nivel de correlación alto $(0,974)$, muy cercano a 1. Consecuentemente es una correlación positiva, significando que a mayor nivel de "Programas de Capacitación para el Recurso Humano", mayor será el crecimiento de las PYMES, dando cumplimiento en la selección de las variables. Respecto a la significación, con resultante de 0,000, hace que se cumpla la condición de significancia inferior a 0,05 , inclusive menor a 0,01 , indicando que la correlación establecida es cierta. Consecuentemente, se rechaza la hipótesis nula y se acepta la hipótesis alternativa.

Al demostrar el resultado de la hipótesis especifica 3, se comprueba que existe un nivel de correlación alta $(0,957)$, próximo a 1 . Esto expresa una correlación positiva, lo que significa que a mayor nivel de innovación de los procesos administrativos, mayor será el crecimiento de las PYMES, así se valida el acierto del escogimiento de las variables. La significación es de 0,000, haciendo que la condición del grado de significancia sea menor a 0,05 , por debajo de 0,01 , corroborando que la correlación es cierta, rechazándose la hipótesis nula y aceptando la hipótesis alternativa.

\section{Nivel de aplicación de estrategias y herramientas}

De las dos variables y las seis dimensiones, tomadas para el estudio de medición del nivel aplicación de estrategias y herramientas de administración en las PYMES hoteleras de Manabí (ver mapa de variables), una vez que se tabularon y procesaron los datos en el programa estadístico SPSS 21, se obtuvieron los siguientes resultados.

Tabla 5: Resultado de la dimensión Prácticas de Marketing

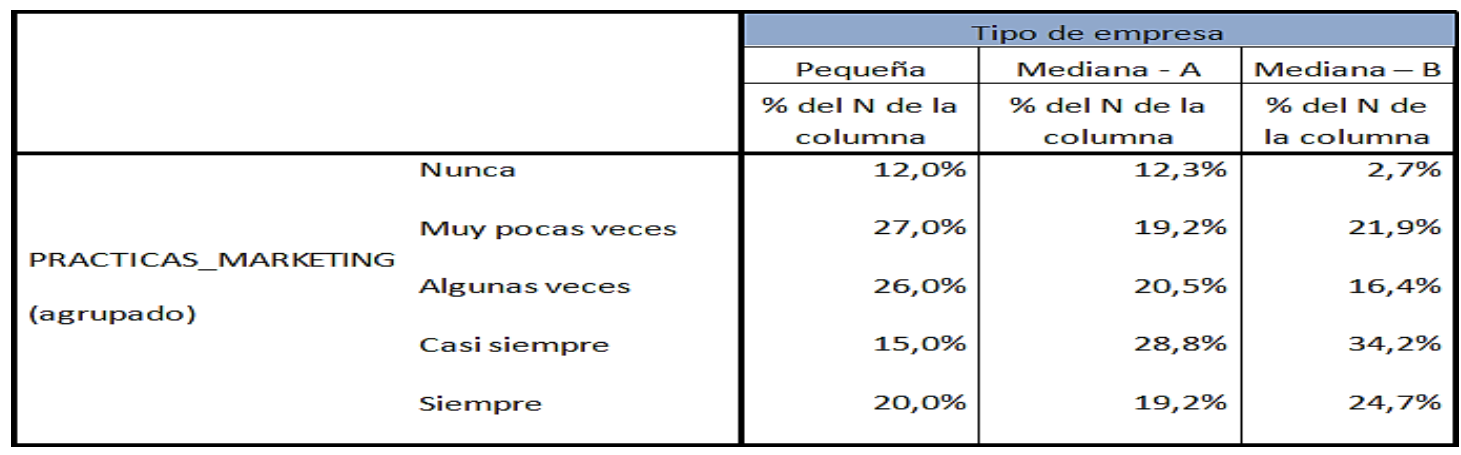

Fuente: Encuestas aplicadas a las PYMES Turísticas Hoteleras de Manabí - Análisis Software SPSS 21 Elaboración: Autores. 
De acuerdo a los resultados de la tabla 5, se demuestra que la aplicación de las prácticas de marketing se manifiestan en mayor proporción en la opción "algunas veces" en las empresas pequeñas $(26,0 \%)$ y en las medianas tipo A $(28,8 \%)$ en la opción "casi siempre", de la misma manera se presentan en mayor proporción en las empresas medianas tipo B (34,2\%).

Tabla 6: Resultados de la dimensión programas de capacitación

\begin{tabular}{|c|c|c|c|c|}
\hline & & \multicolumn{3}{|c|}{ Tipo de empresa } \\
\hline & & Pequeña & Mediana - A & $\begin{array}{c}\text { Mediana - } \\
\text { B }\end{array}$ \\
\hline & & $\begin{array}{c}\% \text { del } \mathrm{N} \text { de la } \\
\text { columna }\end{array}$ & $\begin{array}{l}\% \text { del } \mathrm{N} \text { de la } \\
\text { columna }\end{array}$ & $\begin{array}{l}\% \text { del } \mathrm{N} \text { de } \\
\text { la columna }\end{array}$ \\
\hline \multirow{5}{*}{$\begin{array}{l}\text { PROGRAMAS_CAPACITACION } \\
\text { (agrupado) }\end{array}$} & Nunca & $13,0 \%$ & $5,5 \%$ & $4,1 \%$ \\
\hline & Muy pocas veces & $18,0 \%$ & $11,0 \%$ & $8,2 \%$ \\
\hline & Algunas veces & $26,0 \%$ & $15,1 \%$ & $16,4 \%$ \\
\hline & Casi siempre & $23,0 \%$ & $38,4 \%$ & $35,6 \%$ \\
\hline & Siempre & $20,0 \%$ & $30,1 \%$ & $35,6 \%$ \\
\hline
\end{tabular}

Fuente: Encuestas aplicadas a las PYMES Turísticas Hoteleras de Manabí - Análisis Software SPSS 21 Elaboración: Autores.

Respecto a la dimensión sobre la aplicación de programas de capacitación (tabla 6), se presentan en mayor proporción en la opción "algunas veces" las respuestas de las empresas pequeñas (26\%), mientras que la opción "casi siempre" presentan valores similares en las empresas medianas tipo A $(38,4 \%)$ y medianas tipo B $(35,6 \%)$.

Tabla 7: Resultados de la dimensión de procesos administrativos

\begin{tabular}{|c|c|c|c|c|}
\hline & & \multicolumn{3}{|c|}{ Tipo de empresa } \\
\hline & & Pequeña & Mediana-A & Mediana-B \\
\hline & & $\begin{array}{l}\% \text { del N de la } \\
\text { columna }\end{array}$ & $\begin{array}{l}\% \text { del } \mathrm{N} \text { de } \\
\text { la columna }\end{array}$ & $\begin{array}{l}\% \text { del } \mathrm{N} \text { de la } \\
\text { columna }\end{array}$ \\
\hline \multirow{5}{*}{$\begin{array}{l}\text { INNOVACION_PROCESOS } \\
\text { (agrupado) }\end{array}$} & Nunca & $8,0 \%$ & $2,7 \%$ & $2,7 \%$ \\
\hline & Muy pocas veces & $15,0 \%$ & $12,3 \%$ & $9,6 \%$ \\
\hline & Algunas veces & $25,0 \%$ & $19,2 \%$ & $13,7 \%$ \\
\hline & Casi siempre & $19,0 \%$ & $34,2 \%$ & $30,1 \%$ \\
\hline & Siempre & $33,0 \%$ & $31,5 \%$ & $43,8 \%$ \\
\hline
\end{tabular}

Fuente: Encuestas aplicadas a las PYMES Turísticas Hoteleras de Manabí - Análisis Software SPSS 21 Elaboración: Autores. 
De la misma forma la dimensión sobre la innovación de los procesos administrativos (tabla 7), se presentan en mayor proporción en la opción "siempre" en las empresas pequeñas $(33,0 \%)$, mientras que la opción "casi siempre" se presenta en las empresas medianas tipo A $(34,1 \%)$ y en las medianas tipo B la opción "siempre” (43,8\%).

Tabla 8: Resultados de la dimensión de participación de mercado

\begin{tabular}{|c|c|c|c|c|}
\hline & & \multicolumn{3}{|c|}{ Tipo de empresa } \\
\hline & & Pequeña & Mediana - A & Mediana - B \\
\hline & & $\begin{array}{c}\% \text { del N de la } \\
\text { columna }\end{array}$ & $\begin{array}{l}\% \text { del } \mathrm{N} \text { de } \\
\text { la columna }\end{array}$ & $\begin{array}{c}\% \text { del N de la } \\
\text { columna }\end{array}$ \\
\hline \multirow{5}{*}{$\begin{array}{l}\text { PARTICIPACION_MERCADO } \\
\text { (agrupado) }\end{array}$} & Nunca & $0,0 \%$ & $0,0 \%$ & $0,0 \%$ \\
\hline & Muy pocas veces & $16,0 \%$ & $5,5 \%$ & $6,8 \%$ \\
\hline & Algunas veces & $22,0 \%$ & $23,3 \%$ & $12,3 \%$ \\
\hline & Casi siempre & $25,0 \%$ & $32,9 \%$ & $35,6 \%$ \\
\hline & Siempre & $37,0 \%$ & $38,4 \%$ & $45,2 \%$ \\
\hline
\end{tabular}

Fuente: Encuestas aplicadas a las PYMES Turísticas Hoteleras de Manabí - Análisis Software SPSS 21 Elaboración: Autores.

Por otro lado en la dimensión participación de mercado (tabla 8), se manifiestan en mayor proporción en la opción "siempre": en las empresas pequeñas (37\%), en las empresas medianas tipo A $(38,4 \%)$ y en las medianas tipo B $(45,2 \%)$.

Tabla 9: Resultados de la dimensión ventas

\begin{tabular}{|c|c|c|c|c|}
\hline & & \multicolumn{3}{|c|}{ Tipo de empresa } \\
\hline & & Pequeña & Mediana - A & Mediana - B \\
\hline & & $\begin{array}{c}\% \text { del N de la } \\
\text { columna }\end{array}$ & $\begin{array}{c}\% \text { del } \mathrm{N} \text { de la } \\
\text { columna }\end{array}$ & $\begin{array}{c}\% \text { del } \mathrm{N} \text { de la } \\
\text { columna }\end{array}$ \\
\hline \multirow{5}{*}{$\begin{array}{l}\text { VENTAS } \\
\text { (agrupado) }\end{array}$} & Nunca & $5,0 \%$ & $2,7 \%$ & $1,4 \%$ \\
\hline & Muy pocas veces & $23,0 \%$ & $8,2 \%$ & $2,7 \%$ \\
\hline & Algunas veces & $35,0 \%$ & $15,1 \%$ & $12,3 \%$ \\
\hline & Casi siempre & $18,0 \%$ & $28,8 \%$ & $30,1 \%$ \\
\hline & Siempre & $19,0 \%$ & $45,2 \%$ & $53,4 \%$ \\
\hline
\end{tabular}

Fuente: Encuestas aplicadas a las PYMES Turísticas Hoteleras de Manabí - Análisis Software SPSS 21

Elaboración: Autores. 
Nivel de aplicación de estrategias y herramientas de administración en las PYMES hoteleras de Manabí

Respecto a la dimensión sobre las ventas (tabla 9), se presentan en mayor proporción en la opción "algunas veces" las respuestas de las empresas pequeñas (35\%), mientras que la opción "siempre" presentan en las empresas medianas tipo A $(45,2 \%)$ y medianas tipo B $(53,4 \%)$.

Tabla 10: Rentabilidad

\begin{tabular}{|ll|r|r|r|}
\hline \multirow{2}{*}{} & & \multicolumn{3}{|c|}{ Tipo de empresa } \\
\cline { 3 - 5 } & & \multicolumn{1}{c|}{ Pequeña } & Mediana - A & Mediana - B \\
\cline { 3 - 5 } & $\begin{array}{c}\text { \% del N de la } \\
\text { columna }\end{array}$ & $\begin{array}{c}\text { \% del N de la } \\
\text { columna }\end{array}$ & $\begin{array}{c}\text { \% del N de la } \\
\text { columna }\end{array}$ \\
\hline \multirow{2}{*}{ RENTABILIDAD } & Nunca & $3,0 \%$ & $0,0 \%$ & $0,0 \%$ \\
(agrupado) & Muy pocas veces & $19,0 \%$ & $4,1 \%$ & $1,4 \%$ \\
& Algunas veces & $27,0 \%$ & $12,3 \%$ & $5,5 \%$ \\
& Casi siempre & $28,0 \%$ & $21,9 \%$ & $13,7 \%$ \\
& Siempre & $23,0 \%$ & $61,6 \%$ & $79,5 \%$ \\
\hline
\end{tabular}

Fuente: Encuestas aplicadas a las PYMES Turísticas Hoteleras de Manabí - Análisis Software SPSS 21

Elaboración: Autores.

En cuanto a la dimensión sobre la rentabilidad (tabla 10), se presentan en mayor proporción en la opción "casi siempre" las respuestas de las empresas pequeñas (28\%), mientras que la opción "siempre" se presentan valores máximos en las empresas medianas tipo A $(61,6 \%)$ y medianas tipo B $(79,5 \%)$. 


\section{Discusión}

Si bien se denota cierto nivel de aplicación de estrategias (prácticas de marketing, que alientan la posibilidad de acrecentar las ventas y por ende la participación del mercado, de la mano de la capacitación del recurso humano para mejorar el desempeño del personal), aún queda mucho por desarrollar en las PYMES hoteleras manabitas, debiendo ser consiente el pequeño y mediano empresario que estos resultados, los cuales se están socializando, no les alcanzara para mejorar su competitividad y sostenerse en el tiempo. De igual manera el aprovechamiento de herramientas que brinda la administración moderna, de las cuales se han tomado dimensiones muy básicas (innovación de procesos administrativos con apoyo de las TIC, medir la rentabilidad incorporando el uso de estados financieros, para ver las situación real del negocio en un periodo determinado, y así comprobar si por lo menos se cumple con el punto de equilibrio), reiteran que el proceso de enseñanza/aprendizaje es aun largo.

La presente investigación evidencia la necesidad de que las PYMES del sector hotelero de la provincia de Manabí, deban encaminar sus esfuerzos para satisfacer plenamente a sus clientes; el mercado de servicios cambia continuamente, por esta razón estas unidades de negocio para mantenerse deben adecuarse de manera competitiva a las exigencias del consumidor y los nuevos escenarios que demanda el turismo.

Esta investigación, más allá de provocar un análisis crítico, plantea una discusión formal desde varios frentes, principalmente el académico, empresarial y del estado, quedando clara la necesidad de ejecutar programas de capacitación para las PYMES, por consiguiente, existe un compromiso tripartito de las empresas, el gobierno y la academia. 


\section{Conclusiones}

La investigación revela importantes enfoques diacrónicos de las variables estudiadas, respecto a las teorías de la gestión comercial y el crecimiento, que agrupa a muchos teóricos de la administración clásica, contemporánea y moderna; se demuestra que desde una orientación teórica y práctica la presente investigación, permite obtener nuevos conocimientos e identificar científicamente el problema de las PYMES hoteleras, mismo que evidencia que hay mucho por hacer y contribuir, en la manera en cómo deben ser administradas estas pequeñas y medianas empresas, debiendo cambiar su visión de negocios.

Otro resultado del presente trabajo, es que se logró establecer que la gestión comercial tiene influencia en el crecimiento de las PYMES del sector hotelero de la provincia de Manabí, con base en los resultados de correlación del software estadístico.

Es importante que las PYMES incorporen con más fuerza la gestión comercial, desdoblando acciones que mejoren las prácticas de marketing, que deben ir desde la creación de productos/servicios, precios competitivos y atractivos, concentrándose en desarrollar nuevos nichos de mercado (plaza), publicitando sus propuestas, las cuales deben estar acompañadas de campañas comunicacionales que vinculen las potencialidades de Manabí como destino turístico. Para que el empresario hotelero cumpla sus metas y logre crecer, debe apostar a la capacitación perenne de su recurso humano, modernizando sus procesos administrativos, que no solo mejoren las tareas cotidianas de su empresa, sino para aprovechar la tecnología como un vínculo relacional permanente con los clientes.

Es vital para el sector, sus gremios, las instituciones estatales, universidades y todo organismo vinculado al desarrollo turístico de Manabí, efectuar investigaciones comparativas con otras zonas del país y regiones del mundo, primordialmente con naciones vecinas, a fin de levantar memorias de estudio, cuyos contenidos permitan aprovechar las fortalezas que se tengan, como también minimizar las debilidades. 


\section{Referencias bibliográficas}

Araque, Wilson. (2012). Cuaderno de trabajo: las Pymes y su situación actual. Observatorio de la PYME. Ecuador

Caballero, Miguel Gonzalo; Freijeiro, Ana Belén. (2010). Dirección estratégica de la Pyme: Fundamentos y teorías para el éxito empresarial. Ediciones de la U. Bogotá

Canias, Elkin; Torres, Aníbal. (2013). Gestión empresarial para Pymes. Ed. Uniaula. Medellín

Cazau, P. (2006). Introducción a la investigación en ciencias sociales. Ed. Buenos aires. Tercera edición

Chiavenato, Idalberto. (2011). Administración de recursos humanos: el capital de las organizaciones. McGraw-Hill. México

David, R. Fred. (2013). Conceptos de administración estratégica. Ed. Pearson educación. México

De Borja, Francisco. (2008). Sistematización de la función comercial. Ed. Netbiblo. España

Hernández, R.; Fernández, C.; Baptista, P. (2010). Metodología de la investigación. 5ta Edición. MacGraw-Hill. México

Herrero, P. (2011). Administración, gestión y comercialización en la pequeña y mediana empresa. Editorial Paraninfo

INEC. (2016). Instituto Nacional de Estadísticas y Censos. Ecuador en cifras

Kish, Leslie. (2003). Diseño estadístico para la investigación. Ed. Centro de investigaciones sociológicas. Madrid

Kotler, P. (2005). Nuevos retos de la gestión del marketing. Revista Harvard Deusto. Marketing \& Ventas, Vol. 69 (7-8), pp. 9-12

Krugman, P. (2010). Economía internacional, teoría y política. Ed. Pearson educación. Madrid. 
Dom. Cien., ISSN: 2477-8818

Vol. 4, núm. 1, Enero, 2017, pp. 206-234

Nivel de aplicación de estrategias y herramientas de administración en las PYMES hoteleras de Manabí

Russell, Ackoff. (1979). Prediseñado el futuro. Ed. Limusa. México 
Dom. Cien., ISSN: 2477-8818

Vol. 4, núm. 1, Enero, 2017, pp. 206-234

Nivel de aplicación de estrategias y herramientas de administración en las PYMES hoteleras de Manabí 
Dom. Cien., ISSN: 2477-8818

Vol. 4, núm. 1, Enero, 2017, pp. 206-234

Nivel de aplicación de estrategias y herramientas de administración en las PYMES hoteleras de Manabí 
Dom. Cien., ISSN: 2477-8818

Vol. 4, núm. 1, Enero, 2017, pp. 206-234

Nivel de aplicación de estrategias y herramientas de administración en las PYMES hoteleras de Manabí 
Dom. Cien., ISSN: 2477-8818

Vol. 4, núm. 1, Enero, 2017, pp. 206-234

Nivel de aplicación de estrategias y herramientas de administración en las PYMES hoteleras de Manabí 